\title{
Cooperación, creatividad y emprendimiento para el desarrollo de competencias. Una experiencia docente en Periodismo.
}

\author{
Amparo López Meri
}

Universitat Jaume I de Castelló, meri@uji.es

\begin{abstract}
Recent researches show an increase in university students' entrepreneurial intention. Nevertheless, in degrees not many demanded by job market such as Journalism also grows the students' disappointment when they are in the last courses. Entrepreneurship can be an option in these cases. This teaching experience developed in the 4th year of the Degree in Journalism at the University Jaume I of Castellón aims to improve transversal competences, specially teamwork connected to professionally viable projects. To do this, it has used the potential of the cooperative learning and project-based learning with a creative methodology to increase motivation and involve future journalists in their learning process. The results suggest that students are receptive to educational innovations that can improve team discussion. They work comfortably in small groups and cooperate correctly when tasks are planned in detail by the professor. However, they are reticent to work coordinatelly with other teams in the class to achieve common goals, and they have problems in planning when are given freedom of self-management.
\end{abstract}

Keywords: teacher creativity, cooperative learning, project-based learning, entrepreneurship, competences, higher education

\footnotetext{
Resumen

Recientes investigaciones muestran un aumento en la intención emprendedora del alumnado universitario. Sin embargo, en titulaciones con poca demanda laboral como Periodismo también crece el desencanto a medida que avanzan los cursos. El emprendimiento puede ser una opción en estos casos. Esta experiencia docente, desarrollada en $4^{\circ}$ curso del Grado en Periodismo de la Universitat Jaume I de Castelló, pretende mejorar las competencias transversales del alumnado, especialmente el trabajo en equipo vinculado a iniciativas profesionales viables. Para lograrlo, se ha combinado el potencial del aprendizaje cooperativo y el aprendizaje basado en proyectos con una metodología creativa que persigue incrementar la motivación e implicar a los futuros periodistas en su proceso formativo. Los resultados sugieren que el alumnado es receptivo a las innovaciones docentes que enriquecen la discusión en equipo. Trabajan con comodidad en grupos reducidos y cooperan adecuadamente cuando las tareas están muy pautadas por la profesora. Sin embargo, son reticente a coordinarse con
} 
otros equipos de la clase para lograr metas comunes, y presentan carencias en la planificación cuando disponen de plena libertad para autogestionarse.

Palabras clave: creatividad docente, aprendizaje cooperativo, aprendizaje basado en proyectos, emprendimiento, competencias, educación superior

\section{Introducción}

El Espacio Europeo de Educación Superior (EEES) y el mercado laboral demandan graduados formados y efícientes en competencias transversales. Además de adquirir conocimientos teóricos, se valoran sus habilidades para el trabajo en equipo, la comunicación interpersonal, la integración en la sociedad y el pensamiento crítico. El aprendizaje cooperativo es una forma de trabajo en equipo que contribuye al desarrollo de estas competencias (Traver, 2011). Se fundamenta en la construcción colectiva del conocimiento y el desarrollo de habilidades mixtas (personales, sociales y laborales), donde cada participante es responsable de su formación y del aprendizaje del resto de sus compañeros (García y Troyano, 2010). La interacción conlleva un progreso intelectual (Ovejero Bernal, 1990), especialmente cuando el alumnado trabaja en grupos reducidos, donde se sienten entendidos e integrados, por lo que se generan actitudes positivas hacia sus iguales y hacia las tareas de clase, aumentando la motivación individual, la autoestima y el rendimiento académico (Johnson y Johnson, 1989; Markham et al., 2003).

Por otro lado, cada vez más, se anima al profesorado a promover la cultura del emprendimiento en las aulas. La formación de emprendedores es un motor de desarrollo para las universidades (Laukkanen, 2000). La Educación Superior es responsable de identificar y alentar a las personas que pueden ser emprendedores (Hatten, 1993). Algunas investigaciones muestran que la capacidad potencial de emprender de los estudiantes es significativa, aunque todavía es amplia la brecha entre las ideas y los proyectos que acaban materializándose en negocio (Cabana-Villca et al., 2013). En grados como Periodismo, donde se desarrolla esta experiencia docente, se constata un aumento de la disposición del alumnado a participar en iniciativas empresariales, aunque también crece el desencanto a medida que avanzan los cursos (Casero-Ripollés et al., 2016), en parte por las pocas posibilidades de ser contratado al finalizar los estudios. En este sentido, el aprendizaje basado en proyectos puede ser un revulsivo para recuperar la motivación, despertar la creatividad y formar sujetos proactivos, atributos y actitudes propias del emprendedor (Cano et al., 2003; Loli et al., 2010).

El aprendizaje basado en proyectos, ligado al trabajo cooperativo en equipo, puede contribuir a reproducir en el aula el llamado clima de emprendimiento (Krueger y Brazeal, 1994), recreando las condiciones necesarias para influir en la intención emprendedora del alumnado (Morales Parragué, 2009). La simulación de entornos profesionales obliga al alumnado a enfrentarse a problemas de verdad, y eso despierta su creatividad para superar obstáculos. Cuando los estudiantes aprecian que su esfuerzo y sus ideas podrían tener viabilidad empresarial, entienden la utilidad de las tareas académicas. Mientras trabajan en sus proyectos, interiorizan conceptos y perfeccionan sus destrezas. Por eso, el trabajo

(cc) EY-NC-ND 2016, Universitat Politècnica de València 
cooperativo afianza estrategias de aprendizaje de mayor calado (Gil et al., 2006), pues se retienen mejor los contenidos que se debaten oralmente en grupo, y también se incrementa la capacidad crítica, gracias a las controversias y su resolución en equipo (García et al., 2001). El alumnado se integra activamente en su proceso formativo, asumiendo responsabilidades similares a las que adoptaría en su puesto de trabajo (Baena, 2010).

\section{Objetivos}

El principal objetivo de esta experiencia docente es mejorar el trabajo en equipo y el aprendizaje colaborativo en el aula, mediante la simulación de entornos laborales realistas y la aplicación de metodologías creativas, tanto en la fase de formación de los equipos de trabajo como en la fase de generación y jerarquización de ideas.

En segundo lugar, combinando el aprendizaje cooperativo y el aprendizaje basado en proyectos con una metodología creativa, se persigue crear un clima de emprendimiento en el aula y propiciar una mayor implicación del alumnado en su formación.

Estos propósitos genéricos pueden desglosarse en logros más concretos:

- Fortalecer las habilidades para trabajar en grupo: capacidad de negociación, correcta planificación, diálogo, asertividad y empatía para aprender de compañeros con distintos estilos de aprendizaje.

- Formar al alumnado para la incorporación de técnicas de creatividad en sus procesos de aprendizaje.

- Impulsar la cooperación entre personas con distintos estilos de aprendizaje.

- Incorporar valores basados en la igualdad al trabajo colaborativo y al diseño de proyectos profesionales.

- Desarrollar competencias transversales.

- Potenciar la cultura del emprendimiento en el aula.

En otro orden, para mejorar el planteamiento de este proyecto docente en próximos cursos, se contemplan dos objetivos relacionados con la percepción del alumnado:

- Conocer el grado de satisfacción del alumnado en su relación con el equipo de trabajo que se le ha asignado.

- Conocer el grado de satisfacción del alumnado respecto a la aplicación de técnicas de creatividad y la simulación de entornos profesionales realistas en el aula.

\section{Desarrollo de la innovación docente}

La experiencia docente se ha puesto en práctica en el curso 2015-2016, en la asignatura Comunicación para la Igualdad del Grado en Periodismo de la Universitat Jaume I de Castelló, que se imparte en $4^{\circ}$ curso. Se trata de una asignatura de carácter obligatorio con un contenido idóneo para los objetivos de este proyecto. En Comunicación para la Igualdad se desarrolla el análisis crítico del discurso periodístico en relación a la representación de

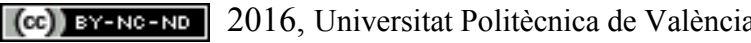

Congreso In-Red (2016) 
colectivos estereotipados o marginados por los medios de comunicación, y se educa al alumnado en valores basados en la igualdad y el respeto a la diversidad. Como alternativas al discurso hegemónico y oficial de los poderes político, financiero y mediático, se estudian medios de comunicación dedicados a las minorías y casos de activismo político en Internet. Precisamente, en las prácticas de la asignatura, el alumnado se enfrenta al reto de ponerse en la piel de un equipo de activistas, pues deben diseñar un proyecto periodístico de monitorización destinado a denunciar abusos de poder por parte de los políticos, gobiernos y otras instituciones. Además, se plantean dos requisitos: se trabaja en grupo y la propuesta del alumnado ha de ser realista y viable desde el punto de vista profesional.

\subsection{Justificación}

Durante el curso, el alumnado observa que los periodistas y los medios de comunicación, en muchas ocasiones, vetan a colectivos minoritarios y contribuyen a la desigualdad social. Con este proyecto, se pretende que el alumnado no incurra en los mismos errores cuando trabaja en grupo, se persigue que sea respetuoso con las aportaciones del resto de compañeros. Asimismo, en tanto que se han de diseñar proyectos profesionales viables, se fomenta la creatividad y el espíritu emprendedor como alicientes para el aprendizaje. Así, en consonancia con las exigencias del Espacio Europeo de Educación Superior (EEES), se trabaja en la formación integral de la persona, pues al tiempo que el alumnado ejerce de periodista comprometido, se le educa en valores y otras competencias transversales que necesitará para adaptarse a las demandas del mercado laboral.

\subsection{Metodología}

\subsubsection{Fase formativa del profesorado}

En el marco del Seminario Permanente de Innovación Educativa (SPIE) de Creatividad en la Docencia de la Universitat Jaume I, en el que participa la profesora de la asignatura Comunicación para la Igualdad del Grado en Periodismo, se organizan diferentes acciones formativas. Durante los cursos 2013-2014 y 2014-2015, el profesorado aprende técnicas para la formación de equipos de trabajo y las prueba en el aula. En el segundo semestre del curso 2014-2015 se da un paso más, por la necesidad de optimizar la generación de ideas y la negociación de propuestas dentro de los equipos de trabajo. Por ello, el profesorado de este SPIE recibe formación específica para la aplicación de técnicas y herramientas de creatividad que implementen estos procesos. El reto para el curso 2015-2016 es poner en práctica alguna de estas técnicas en clase, en un contexto que además propicie la recreación de entornos laborales realistas y el emprendimiento. La experiencia docente que se describe en este artículo es fruto de este reto.

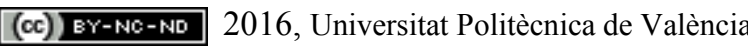




\subsubsection{Fase formativa del alumnado}

Antes de crearse los equipos de trabajo, el alumnado recibe formación en dos sentidos. En primer lugar, para conseguir que los proyectos de clase sean profesionalmente viables, se analizan en el aula ejemplos de plataformas de monitorización como MySociety, Fundación Civio o Ciudadano Inteligente. Así, el alumnado realizó un breve estudio de las iniciativas que estas plataformas desarrollan, se presentó oralmente y se compartieron las principales conclusiones. En segundo lugar, se explicó cómo emplear la técnica creativa del $4 \times 4 \times 4$ para enriquecer la selección y jerarquización de ideas.

\subsubsection{Fase de formación de los equipos de trabajo}

La formación de los equipos se realizó combinando alumnos con diferentes estilos de aprendizaje, a partir de sus respuestas al cuestionario de Jiménez (2006), una adaptación del test elaborado por Ned Herrmann (1989) para desarrollar su Modelo del Cerebro Total. Herrmann describe cuatro cuadrantes cerebrales, cada uno especializado en unas funciones determinadas (Fígura 1). El lóbulo superior izquierdo (cuadrante A) se especializa en el pensamiento lógico-analítico, cuantitativo, matemático y basado en hechos. El lóbulo inferior izquierdo (cuadrante B), se dedica al pensamiento secuencial, organizado, planificado y detallado. El lóbulo inferior derecho (cuadrante C) se asocia al pensamiento emocional, comunicador, sensorial, espiritual y humanístico. Por último, el lóbulo superior derecho (cuadrante D) se basa en el pensamiento conceptual, estratégico, integrador, creativo, espacial y visual.

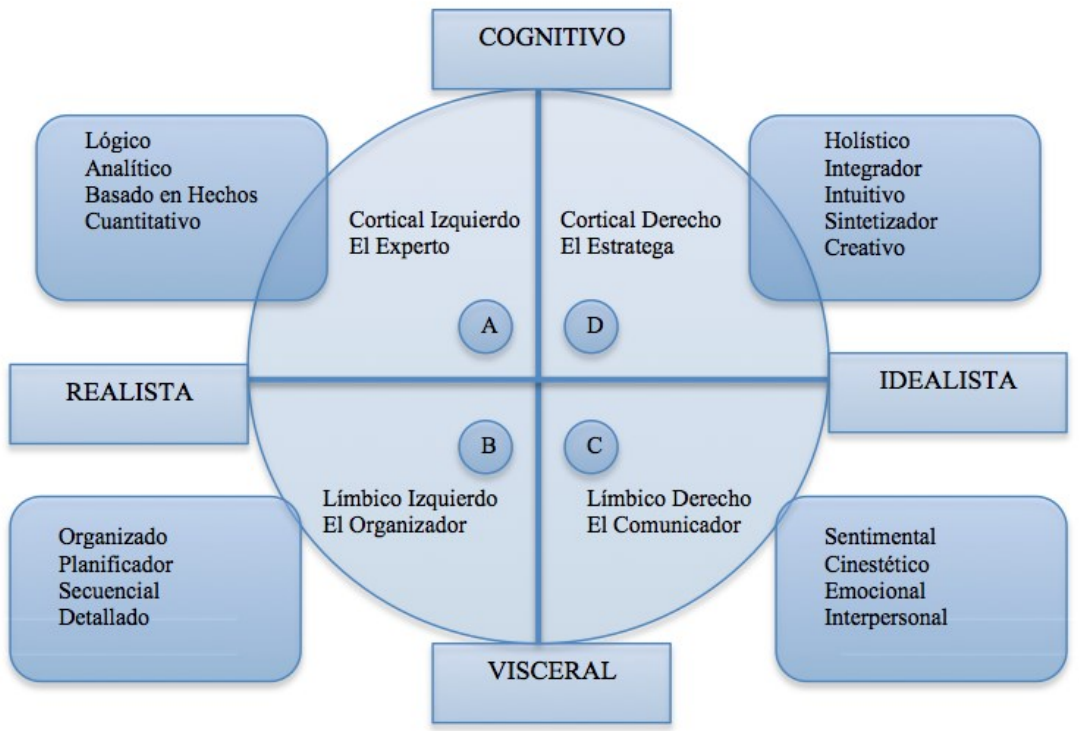

Fig. 1 Modelos de estilos de pensamiento según Herrmann (basado en Cazau, 2004)

(c)) EY-NC-ND 2016, Universitat Politècnica de València

Congreso In-Red (2016) 
Conforme al modelo de Herrmann, a través de un cuestionario de 40 ítems (Tabla 1), es posible detectar los cuadrantes cerebrales que más utiliza cada alumno conforme a cuatro perfiles de pensamiento:

- Pensamiento realista, propio del hemisferio izquierdo (cuadrantes A y B)

- Pensamiento idealista, propio del hemisferio derecho (cuadrantes C y D)

- Pensamiento pragmático (cuadrantes A y D)

- Pensamiento instintivo (cuadrantes B y C)

El alumnado respondió al cuestionario y expresó su opinión, en una escala tipo Likert de 5 puntos (donde 1 es "lo que hago peor" y 5 es "lo que hago mejor"), sobre la percepción que tiene de su grado de cumplimiento de los aspectos asociados a cada cuadrante cerebral.

Tabla 1. Escala de medición para el diagnóstico del Modelo del Cerebro Total

\section{Cuadrante A (superior izquierdo cortical)}

1. Tengo habilidades específicas en el campo de las matemáticas y las ciencias.

2. Pienso que la mejor forma de resolver un problema es siendo analítico.

3. Me inclino hacia la crítica en todos los asuntos.

4. Tengo habilidades para solucionar problemas complejos de manera lógica.

5. Antes de tomar algo como verdadero, lo compruebo, e indago otras fuentes.

6. Tengo capacidad de comprender, manipular números y estadísticas de acuerdo con un fin.

7. Me gusta solucionar problemas inclinándome a conocerlos y buscar mediciones exactas.

8. Tengo capacidad ante los problemas de razonar en forma deductiva, a partir de alguna teoría.

9. Descompongo ante un problema las ideas y las relaciono con la totalidad.

10. Selecciono alternativas sobre la base de la racionalidad y la inteligencia, en oposición al instinto, a la emoción.

Cuadrante B (inferior izquierdo límbico)

11. La planificación y la organización son prioritarias en mis actividades.

12. Es importante para mí tener un lugar para cada cosa y cada cosa en su lugar.

13. Acostumbro escuchar las opiniones de los demás y hacer aclaraciones.

14. Prefiero instrucciones específicas en lugar de aquellas generales que dejan muchos detalles opcionales.

15. Pongo mucha atención en los pequeños detalles o partes de un proyecto.

16. Tengo capacidad de control y dominio de mis emociones cuando elaboro un plan o proyecto.

17. Pienso que trabajar con un método paso a paso es la mejor manera de resolver mi problema.

18. Tengo habilidades específicas en el manejo de auditorio o hablar en público.

19. Formulo métodos o medios para alcanzar un fin deseado antes de pasar a la acción.

20. Tengo la capacidad de coordinar a las personas o de ordenar los elementos para lograr relaciones coherentes y armoniosas.

\section{Cuadrante C (inferior derecho límbico)}

21. Prefiero trabajar en equipo que hacerlo sólo.

22. Es importante para mí estar en muchas oportunidades acompañado.

23. Creo en la trascendencia humana, en algo superior o espiritual.

24. Soy emotivo frente a las situaciones difíciles. 
25. A menudo actúo para solucionar problemas de tipo social.

26. En muchas ocasiones prima más en mis decisiones, lo emotivo que lo lógico y lo racional.

27. Disfruto, observo y me emociono frente a la belleza de la naturaleza.

28. Tengo habilidades para percibir, entender, manipular posiciones relativas de los objetos en el espacio.

29. Utilizo todos mis sentidos con frecuencia para resolver problemas (olfato, vista, gusto, tacto, oído).

30. Tengo la capacidad de desarrollar y mantener buena comunicación con diferentes tipos de personas.

\section{Cuadrante D (superior derecho cortical)}

31. Tengo un interés muy fuerte o talento con la música, la poesía, la escultura. También para pintar, dibujar, esquematizar etc.

32. Tengo la capacidad de razonar en forma avanzada y creativa, siendo capaz de adquirir, modificar y retener conocimientos.

33. Produzco nuevas ideas e innovaciones en mi trabajo.

34. Tengo la capacidad de entender y hacer uso de imágenes visuales y verbales para representar semejanzas y diferencias.

35. Tengo la capacidad de percibir y entender una problemática global sin entrar en el detalle de los elementos que la componen.

36. A menudo mis mejores ideas se producen cuando no estoy haciendo nada en particular.

37. Prefiero ser conocido y recordado como una persona imaginativa y fantasiosa.

38. Frecuentemente me anticipo a la solución de los problemas.

39. Tengo la capacidad de utilizar o comprender objetos, símbolos y señales complejas.

40. Utilizo el juego y el sentido del humor en muchas de mis actividades.

Fuente: Jiménez (2006)

El resultado en cada cuadrante se obtiene al multiplicar por dos los puntos. La dominancia (puntuación superior a los 67 puntos) en uno u otro cuadrante determina diferentes estilos de pensamiento, que derivan en diferentes estilos de aprendizaje:

- Cuadrante A. Las personas con dominancia en A (hemisferio superior izquierdo) son analíticas, lógicas, se guían por teorías, proceden por hipótesis, prefieren la precisión y tienen facilidad para las matemáticas. Son personas intelectualmente brillantes, frías, críticas, competitivas e individualistas.

- Cuadrante B. Las personas con dominancia en B (hemisferio inferior izquierdo) son introvertidas, emotivas, conservadoras, metódicas y trabajadoras. Se les da bien planificar y estructurar, y tienen dotes para la organización y el liderazgo.

- Cuadrante C. Las personas con dominancia en C (hemisferio inferior derecho) son extrovertidas, emotivas, espontáneas, habladoras y espirituales. Reaccionan mal a las críticas negativas, pero tienen competencias para las relaciones interpersonales, el diálogo, la enseñanza y el trabajo en equipo.

- Cuadrante D. Las personas con dominancia en D (hemisferio superior derecho) son independientes, innovadoras, creativas y emprendedoras. Tienen sentido del humor, les gusta el riesgo e imaginan y visualizan el futuro con facilidad.

(c)) EY-NC-ND 2016, Universitat Politècnica de València

Congreso In-Red (2016) 
No obstante, la dominancia simple (solo en un cuadrante) no es habitual. Es frecuente presentar dominancias en dos o tres cuadrantes simultáneamente (Estrada et al., 2014). De este modo, al combinar alumnos con diferentes perfiles de dominancia para formar los grupos de trabajo, se perseguía que los equipos fueran más completos (Herrmann, 1989), que los estudiantes se complementaran según sus características y capacidades individuales, para fomentar la pluralidad de enfoques y enriquecer la negociación de propuestas.

En total, para el desarrollo de esta iniciativa han participado 48 estudiantes, 27 mujeres y 21 hombres, con edades comprendidas entre los 21 y los 34 años, distribuidos en dos grupos de prácticas de 24 personas (Prácticas A y Prácticas B). Cada grupo de prácticas debía elegir y diseñar un único proyecto de monitorización compartido. Para realizar propuestas y organizar las tareas se crearon equipos más reducidos, en total 6 equipos de 8 personas (combinando estratégicamente alumnos con diferentes perfiles de dominancia cerebral). En concreto, se realizaron las siguientes combinaciones: dos grupos $\mathrm{A}+\mathrm{B}$ (con dominancia primaria en $\mathrm{A}$ y $\mathrm{B}$ ), dos grupos $\mathrm{B}+\mathrm{D}$, un grup $\mathrm{A}+\mathrm{B}+\mathrm{D}$ y un grupo $\mathrm{B}+\mathrm{C}+\mathrm{D}$.

\subsubsection{Fase de diseño y elección del proyecto de monitorización al poder}

La presentación de ideas para elegir el proyecto periodístico de monitorización se secuenció en fases con la técnica creativa del $4 \times 4 \times 4$. Se trata de una técnica grupal de producción y priorización de ideas, orientada a mejorar la comunicación y la cohesión en el equipo. Esta técnica se inicia con una fase individual de brainstorming, que después se completa en sucesivas etapas, doblando el número de participantes en cada escalón.

- En la fase individual, cada alumno escribe en un papel cuatro propuestas o argumentos acerca del foco creativo, en este caso, sobre posibles temas o instituciones a las que monitorizar.

- En la segunda etapa se trabaja en parejas. Cada pareja cuenta con ocho ideas (cuatro por cada individuo) y debe llegar a un acuerdo para seleccionar las cuatro que considere esenciales, que pasarán a la siguiente fase. El objetivo es enriquecer su aportación. Pueden modificar las propuestas originarias para mejorarlas.

- En la tercera fase se trabaja en grupos de cuatro personas (se unen dos parejas de la fase anterior, cada pareja con cuatro ideas). De nuevo, el grupo parte con ocho propuestas y debe descartar la mitad. La jerarquización y selección implica la negociación entre los componentes del grupo. Deben mejorar las propuestas antes de pasar a la siguiente etapa. Entran en juego capacidades y competencias relevantes para el trabajo en equipo (argumentación, comunicación, liderazgo, asertividad, empatía, inteligencia emocional).

- El mismo proceso se repite en la última fase, esta vez en grupos de ocho personas (se unen dos dobles parejas de la fase anterior, cada doble pareja con cuatro propuestas). De nuevo, tienen ocho posibilidades y han de seleccionar cuatro. Se entiende que las cuatro ideas finales de cada equipo serán las más interesantes de todas las producidas anteriormente.

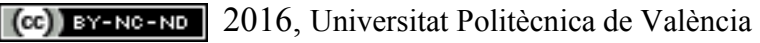


Durante el desarrollo de las distintas fases, la profesora asumió el papel de coordinadora, pautó las normas de funcionamiento y asignó los tiempos de negociación, entre 5 y 10 minutos por fase. En las tres primeras fases, trabajaron juntos estudiantes con la misma dominancia cerebral. La mezcla con alumnos con diferentes dominancias (diferentes estilos de aprendizaje) se realizó en la fase final, al conformar los equipos definitivos. Se unió a personas con diferentes capacidades individuales para enriquecer el debate y las propuestas. Respecto a las instrucciones concretas, se le pidió al alumnado que, además de proponer temas susceptibles de ser monitorizados, anotaran también los argumentos a favor de cada propuesta. En relación a las propuestas finales, cada equipo dispuso de una semana para investigar su viabilidad, incluso para descartar algunas ideas. Tras este período, cada grupo defendió sus mejores aportaciones oralmente, para convencer al resto de equipos antes de la votación final (Figura 2). El objetivo era elegir un único proyecto, el más votado (un voto por alumno). Los diferentes equipos de trabajo (6 en el total, 3 en cada grupo de prácticas) colaboraron para sacar adelante el proyecto compartido.

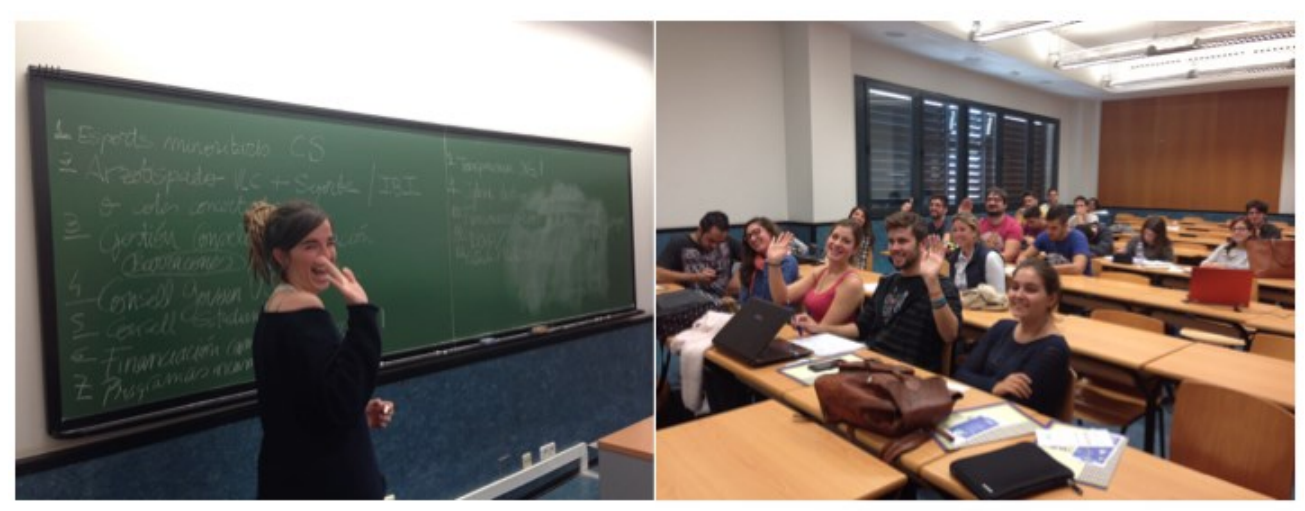

Fig. 2 Imágenes del alumnado durante la votación para seleccionar el proyecto definitivo

\subsubsection{Fase de valoración por parte del alumnado}

El grado de satisfacción del alumnado se ha evaluado con la escala de Viles et al. (2013). Cada alumno ha expresado su grado de acuerdo en relación a 23 afirmaciones relacionadas con el funcionamiento de su equipo durante la fase de propuesta de ideas y selección del proyecto (Tabla 2), mediante una escala tipo Likert de 5 puntos (donde 1 es "totalmente en desacuerdo" y 5 es "totalmente de acuerdo").

Tabla 2. Escala de medición para evaluar la satisfacción respecto al trabajo en equipo

\begin{tabular}{|l|}
\hline \multicolumn{1}{|c|}{ PARTICIPACIÓN EN LA TOMA DE DECISIONES } \\
\hline $\begin{array}{l}\text { 1. Se han establecido normas internas que han facilitado la propuesta de temas de monitorización y } \\
\text { su discusión }\end{array}$ \\
\hline $\begin{array}{l}\text { 2. Todos los miembros han participado en la propuesta de temas para el proyecto de } \\
\text { monitorización. }\end{array}$ \\
\hline 3. Las decisiones en el equipo se han tomado teniendo en cuenta la opinión de todos los miembros. \\
\hline
\end{tabular}

\section{(cc) EY-NC-ND 2016, Universitat Politècnica de València}

Congreso In-Red (2016) 
Cooperación, creatividad y emprendimiento para el desarrollo de competencias. Una experiencia

\section{GESTIÓN DE CONFLICTOS}

4. Las discrepancias en el equipo han permitido considerar nueva ideas o nuevos puntos de vista.

5. No ha habido conflictos de tipo interpersonal y si los ha habido, se han resuelto sin que nadie se haya sentido perjudicado.

\section{RESOLUCIÓN DE PROBLEMAS}

6. Se ha utilizado documentación, información, datos... o se ha seguido algún tipo de método para la selección del tema-proyecto de monitorización.

7. Se ha potenciado la creatividad para llegar a las propuestas de monitorización y resolver el ejercicio.

\section{COOPERACIÓN}

8. Las tareas concretas que cada miembro del equipo tenía que realizar han estado claras desde el inicio, tanto en contenido como en plazo.

9. Tus compañeros de grupo han contribuido tal y como se estableció y como ha sido necesario.

10. Ha existido colaboración entre los miembros del equipo (hemos compartido información y dificultades, nos hemos ayudado...)

\section{LIDERAZGO (PORTAVOZ-PORTAVOCES)}

11. El portavoz del equipo ha sido aceptado por todos los miembros del equipo.

12. El portavoz del equipo ha coordinado las actividades del equipo.

\section{COMUNICACIÓN INTERNA}

13. El trabajo del equipo ha transcurrido en un ambiente de confianza.

14. A pesar de las diferencias, ha existido un ambiente de respeto entre todos.

15. En general, la comunicación ha sido buena entre los miembros del equipo.

\section{FEEDBACK}

16. Los objetivos de la elaboración de un proyecto de monitorización se han transmitido bien y han sido comprendidos.

17. El profesor ha facilitado los recursos necesarios (instrucciones, información, tiempo para trabajar en el aula...)

18. Las tareas concretas (propuesta y selección de temas, documentación, monitorización, redacción, elaboración del trabajo...) han estado claras tanto en contenido como en plazo.

19. Los criterios de evaluación se han transmitido bien al equipo y han sido comprendidos al inicio del proyecto de monitorización.

20. Como equipo, hemos tenido acceso a la información que hemos necesitado sobre cómo desarrollar este ejercicio.

21. En caso de necesidad, el equipo ha podido comunicarse fácilmente con la profesora.

22. Como equipo, hemos recibido información durante la elaboración del ejercicio (orientación para superar dificultades sobrevenidas, alternativas, aspectos positivos, aspectos mejorables...)

23. El trabajo en el proyecto ha sido de alguna manera valorado públicamente al resto de la clase (defensa de propuestas en clase, votación conjunta en público, valoración oral de la profesora...)

Fuente: Viles et al. (2013) 


\section{Resultados}

\subsection{Aproximación a los estilos de aprendizaje del alumnado de Periodismo}

Los datos obtenidos mediante el cuestionario para detectar las dominancias cerebrales del alumnado se han analizado con el programa estadístico SPSS. Tras su estudio, se detectan aspectos interesantes (Tabla 3). Casi el $69 \%$ del alumnado presenta dominancia primaria (puntuación superior a 67) en el cuadrante B (hemisferio inferior izquierdo). Por lo tanto, un grupo numeroso de alumnos se considera metódico y especialmente capacitado para la organización y la planificación. También destaca el porcentaje registrado en el cuadrante D (hemisferio superior derecho). Así, el 56\% de los participantes tiene la percepción de ser competente para la innovación, la creatividad y el emprendimiento. Asimismo, resulta llamativo que sea menor la dominancia en C (37\%), puesto que es un cuadrante relacionado con características y competencias asociadas a titulaciones como Periodismo. En cambio, la dominancia en A es significativa (casi el 46\%) si se tiene en cuenta que es un cuadrante asociado a titulaciones técnicas. Al respecto, resulta curioso que en esta promoción de Periodismo, en términos globales, se consideran más analistas y lógicos (cuadrante A) que dotados para las relaciones interpersonales, el trabajo en equipo y la comunicación (cuadrante C), si bien es cierto que estas capacidades o competencias juegan un papel destacado como dominancia secundaria (el 60\% del alumnado tiene dominancia secundaria en el cuadrante $C$, entre 34 y 66 puntos). También se detecta que más de la mitad de los participantes presenta dominancia mixta, es decir, utilizan con intensidad ambos lados del cerebro (cuadrantes B y D).

Tabla 3. Dominancias cerebrales del alumnado de $4^{\circ}$ de Periodismo (curso 2015-2016)

\begin{tabular}{|c|c|c|c|c|}
\hline Cuadrantes & $\mathbf{A}$ & B & $\mathbf{C}$ & $\mathbf{D}$ \\
\hline Media & 1,54 & 1,31 & 1,65 & 1,44 \\
\hline Desv. Tipica & 0,504 & 0,468 & 0,526 & 0,501 \\
\hline Dominancias & $\%$ (muestra) & $\%$ (muestra) & $\%$ (muestra) & $\%$ (muestra) \\
\hline Terciaria $(0-33)$ & $0 \%(0)$ & $0 \%(0)$ & $2,1 \%(1)$ & $0 \%(0)$ \\
\hline Secundaria (34-66) & $54,2 \%(26)$ & $31,3 \%(15)$ & $60,4 \%(29)$ & $43,8 \%(21)$ \\
\hline Primaria $(67-100)$ & $45,8 \%(22)$ & $68,8 \%(33)$ & $37,5 \%(18)$ & $56,3 \%(27)$ \\
\hline \multicolumn{3}{|c|}{ Dominancias primarias } & $\%$ & Muestra \\
\hline \multicolumn{3}{|c|}{ Sin dominancias primarias } & $12,50 \%$ & 6 \\
\hline \multicolumn{3}{|c|}{ Dominancia hemisferio izquierdo } & $35,42 \%$ & 17 \\
\hline \multicolumn{3}{|c|}{ Dominancia hemisferio derecho } & $27,08 \%$ & 13 \\
\hline \multicolumn{3}{|c|}{ Dominancia mixta (hemisferio izquierdo y derecho) } & $56,25 \%$ & 27 \\
\hline
\end{tabular}

Fuente: Elaboración propia

(cc) EY-NC-ND 2016, Universitat Politècnica de València

Congreso In-Red (2016) 


\subsection{Diseño del proyecto profesional}

Cada grupo de prácticas, formado por 24 alumnos, seleccionó y diseñó un proyecto de monitorización, que representó el $20 \%$ de la nota de la asignatura Comunicación para la Igualdad. La fase de propuesta y selección de ideas resultó muy productiva y motivadora, en parte, por la formación de los equipos conforme a criterios novedosos para el alumnado, y también por la dinámica que supuso la aplicación de la técnica $4 \times 4 \times 4$. Participaron muy activamente y se estimuló su lado creativo. No obstante, el entusiasmo inicial descendió a medida que debían asumir más responsabilidades y trabajar de manera autónoma.

El grupo Prácticas A eligió investigar la dejadez del Gobierno valenciano respecto a las infraestructuras educativas. En concreto, se propuso realizar un mapa interactivo de los centros de Educación Primaria y Secundaria de la Comunidad Valenciana que todavía tienen barracones. A partir del diálogo entre los diferentes equipos de trabajo surgieron ideas interesantes, como realizar una ficha desplegable de cada centro educativo con los principales datos y grabar entrevistas en vídeo. Sin embargo, pese al buen arranque y el interés del alumnado, los equipos de trabajo no se autogestionaron correctamente, en parte por la ausencia de plazos periódicos de entrega de tareas (únicamente se estableció un plazo para la entrega del producto final acabado). Ante las dificultades para acabar a tiempo, se vieron obligados a sustituir el mapa interactivo por una serie de reportajes. Los equipos compartieron documentación, pero confeccionaron sus reportajes sin interactuar con el resto de equipos.

Por su parte, el grupo Prácticas B decidió estudiar los derechos de los consumidores y usuarios en diferentes ámbitos (restauración, asistencia a espectáculos, telefonía móvil, relación con las fuerzas y cuerpos de seguridad del Estado), con el objetivo de denunciar abusos y aportar recomendaciones a la ciudadanía. En este caso, tampoco funcionó la autogestión sin plazos de entrega periódicos ni la coordinación entre equipos. Finalmente, se repartieron los temas y cada equipo elaboró su propio trabajo.

Si se relaciona el trabajo en equipo durante la propuesta de ideas con los estilos de aprendizaje dominantes, cabe destacar que los dos grupos que presentaron las ideas más originales, a juicio de la profesora, responden a las combinaciones A+D (Prácticas A) y $\mathrm{A}+\mathrm{B}+\mathrm{D}$ (Prácticas B). Por lo tanto, podría concluirse que la dominancia en el cuadrante cerebral $\mathrm{D}$, asociada al pensamiento intuitivo y creativo, en coordinación con la dominancia en el cuadrante cerebral A, ligada al pensamiento analítico y lógico, resulta eficaz para generar propuestas de calidad. Además, si se atiende al perfil concreto de las 16 personas que integran estos dos equipos, se aprecia que, aunque suelen obtener más puntos en el cuadrante cerebral D, en el $43,7 \%$ de los casos se comparte dominancia primaria en B, C y D (7 personas obtienen más de 66 puntos en estos tres cuadrantes). A partir de este dato, podría entenderse que las mejores propuestas de la clase surgieron de personas organizadas, emocionales, con facilidad para la comunicación interpersonal y con tendencia al pensamiento creativo. Respecto a sus calificaciones, la mayoría se sitúa en torno al notable. Así, el 68,7\% obtiene una nota entre el 7 y el 8,5 (sobre 10). En este sentido, cabe matizar que otros grupos consiguieron notas más altas. Por eso, en este caso, no puede concluirse que exista correlación entre creatividad y resultados. Por lo tanto, en próximos cursos, sería

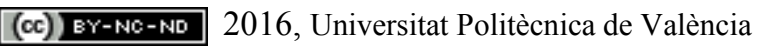


necesario profundizar en el diagnóstico de otros factores que, combinados con la creatividad, puedan mejorar el trabajo final del alumnado.

\subsection{Evaluación del grado de satisfacción del alumnado}

En términos globales, según las respuestas al cuestionario de Viles et al. (2013), la mayoría está conforme con las dinámicas generadas dentro de su equipo de trabajo. El grado de satisfacción es bueno respecto a la participación, la gestión de conflictos y la resolución de problemas dentro de los equipos (más del 60\% a favor), y elevado en relación a la comunicación interna y el feedback con la profesora (entre el $70 \%$ y el $80 \%$ a favor). En este sentido, el 70\% del alumnado considera que la comunicación interna ha sido buena, y el $77 \%$ defiende que ha existido un ambiente de respeto. Además, el $66 \%$ está bastante o totalmente de acuerdo en que la toma de decisiones se ha realizado considerando la opinión de los ocho componentes del equipo, y el 64\% está bastante o totalmente de acuerdo en que las discrepancias han permitido considerar nuevas ideas o nuevos puntos de vista.

Sin embargo, el grado de satisfacción sobre la cooperación para la elaboración de tareas concretas es moderado. El $52 \%$ se muestra indiferente o en desacuerdo en que sus compañeros han contribuido al trabajo como se estableció y como ha sido necesario, y el $42 \%$ duda de que haya existido una verdadera colaboración entre los miembros del equipo.

En relación a la aplicación de técnicas creativas, el $60 \%$ está de acuerdo en que se ha potenciado la creatividad para debatir propuestas y resolver el ejercicio (nadie está totalmente en desacuerdo con esta afirmación). Junto al cuestionario, se incluyó un apartado para comentarios del alumnado. Estos ejemplos resumen sus valoraciones:

- "La técnica creativa $4 \times 4 \times 4$ es interesante, si bien al formar los grupos de forma digamos aleatoria, ello no contribuyó a una comunicación fluida, al menos en mi grupo (...) me parece que podría repetirse en años próximos. La exposición de las ideas de los equipos y la votación resultaron positiva, y considero que, además, se presentaron proyectos interesantes."

- "Ha sido una actividad bastante amena y gratificante. El ejercicio del 4x4x4 me pareció un método curioso para llegar a obtener dos grandes bosquejos de proyectos finales. Es algo fresco y diferente, donde, en mi opinión, el alumnado en cierta forma pone mayor interés y se involucra más desde el primer momento."

- "Separarnos según nuestro tipo de "cerebro" fue muy interesante, pero a la vez pudo resultar perjudicial a la hora de juntarnos con compañeros que no saben trabajar en grupo."

- "Sobre el proyecto final, es difícil gestionar un proyecto con un equipo humano tan amplio. Considero que la propuesta inicial resultaba para todos ilusionante, si bien no se pudo llevar a la práctica finalmente, en parte porque una mayoría de la clase quizá no confiaba en la posibilidad de organizar un proyecto con tantas personas, y también por el hecho de que no se fijaron hitos por así decirlo, en el sentido de que una vez establecidas las funciones de cada uno para el proyecto no 
se señalaron fechas concretas para completar determinadas partes del trabajo, por lo que hubo dejadez por parte de todos nosotros."

Por otro lado, si se relaciona el grado de satisfacción con las dominancias cerebrales, se observan matices interesantes que pueden resultar útiles para mejorar el enfoque de esta actividad. En concreto, a modo de ejemplo, se ha fijado la atención en el grado de satisfacción de las personas que simultáneamente presentan dominancia primaria en los cuadrantes cerebrales B, C y D, por ser una de las combinaciones que mejor funcionó en la fase inicial de propuesta de ideas. Al respecto, cerca del $50 \%$ de las personas en esta circunstancia cuestiona uno o más aspectos relacionados con el trabajo en equipo, especialmente la falta de comunicación interna y la falta de un verdadero ambiente de confianza. El mismo porcentaje se muestra escéptico respecto a que se haya potenciado la creatividad.

\section{Conclusiones}

El principal objetivo de esta experiencia docente consistía en mejorar el trabajo en grupo y el aprendizaje colaborativo a través de la simulación de entornos laborales realistas y la aplicación de metodologías creativas. En este sentido, el alumnado reaccionó con ilusión a las innovaciones introducidas tanto en la fase de formación de los equipos como en la fase de generación y jerarquización de propuestas. Sin embargo, se han detectado algunas carencias respecto al trabajo en equipo y la cooperación.

En general, el alumnado ha demostrado ser receptivo a introducir novedades. También reaccionan con entusiasmo si los proyectos se vinculan al mundo profesional, especialmente cuando las tareas a emprender están pautadas por la profesora. En cambio, responden mal ante demasiada autonomía. Sin plazos de entrega períodicos y evaluables se sienten perdidos, posponen la toma de decisiones y esperan a que otras personas den el primer paso. Por eso, las fases iniciales de la actividad se desarrollaron con normalidad, pues estaban guiadas por la profesora, pero descendió el ritmo de trabajo y la implicación a medida que crecieron las responsabilidades y la libertad de los equipos para su autogestión.

Por otro lado, se constata que el alumnado trabaja cómodamente en grupos reducidos, como es el caso, con equipos de ocho personas. Sin embargo, falla la comunicación y la cooperación intergrupos. Por lo tanto, les resulta complicado coordinarse con otros equipos para alcanzar metas globales (metas compartidas por toda la clase), pero se comunican bien con los compañeros de su propio grupo. No obstante, según la encuesta de satisfacción, aunque consideran que la comunicación interna fue buena, son menos optimistas respecto a la cooperación. Se intuye que pudo haber cierto desequilibrio dentro de los equipos en cuanto a las aportaciones individuales.

Al respecto, para subsanar las carencias descritas y mejorar la cooperación, sería recomendable repetir proyectos docentes similares pero reduciendo el grado de autogestión del alumnado y pautando la entrega periódica de tareas evaluables, para tratar de favorecer la planificación y distribución interna de tareas. Asimismo, resultaría interesante exportar 
esta experiencia a otros grados, aunque parece aconsejable ceñirse a la cooperación intragrupos y eliminar las metas globales de la clase.

Por otra parte, en relación a las dominancias cerebrales o estilos de aprendizaje detectados en la clase (ver Tabla 3), se aprecian algunas tendencias y contradicciones. Resulta llamativo que el alumnado muestre carencias en la planificación para desarrolar el producto final, cuando la dominancia cerebral más destacada se registra en el cuadrante B, relacionado precisamente con competencias como la organización y la planificación (ver Figura 1). Podría inferirse que, aunque se perciben como personas metódicas, no logran organizarse adecuadamente cuando han de autogestionar trabajos en equipo sin plazos de entrega periódicos.

Asimismo, se detecta que el perfil del alumnado de Periodismo puede estar cambiando. La titulación de Periodismo tradicionalmente se ha vinculado a características, capacidades y competencias propias del cuadrante cerebral C, tales como el diálogo, la comunicación, las relaciones interpersonales o el trabajo en equipo. Sin embargo, el alumnado de esta promoción obtiene más puntos en los cuadrantes $\mathrm{B}$ y $\mathrm{D}$, si bien es cierto que un $31 \%$ de los casos presenta simultáneamente dominancia primaria en $\mathrm{C}$ (más de 66 puntos en el cuadrante $\mathrm{C}$, pero menos puntos en $\mathrm{C}$ que en $\mathrm{B}$ o D), y un $60 \%$ tiene dominancia secundaria en $\mathrm{C}$ (entre 34 y 66 puntos en el cuadrante cerebral C).

Respecto a la valoración del alumnado, pese a que la tónica general alcanza un buen grado de satisfacción, si se atiende a grupos con rasgos concretos (combinaciones concretas de dominancias cerebrales), se aprecian discrepancias más acusadas. De hecho, algunos equipos se muestran bastante disconformes respecto a las dinámicas y aportaciones de sus propios compañeros. Ante este indicio, en futuras investigaciones, resultaría aconsejable analizar con más detalle la vinculación entre dominancias cerebrales y grado de satisfacción. Asimismo, no se ha detectado correlación entre creatividad y calificaciones, pero sería interesante seguir profundizando en este ámbito. En este sentido, sería necesario realizar una evaluación inicial y una evaluación final, para poder examinar con mayor fiabilidad la contribución de las actividades desplegadas en el aula al proceso de enseñanzaaprendizaje, especialmente en relación a las acciones dedicadas a potenciar la creatividad. Además, pese a las dificultades surgidas, parece razonable seguir apostando por la combinación de alumnos con diferentes estilos de pensamiento para formar grupos de trabajo más ricos y complementarios.

\section{Referencias}

BAENA, V. (2010). "Innovación docente e identificación de inhibidores del aprendizaje en el área de empresa. Una propuesta metodológica”, Espiral-Cuadernos del Profesorado, vol. 3, nº, pp. 3-14.

CABANA-VILLCA, R., CORTES-CASTILLO, I., PLAZA-PASTEN, D., CASTILLO-VERGARA, M., y ALVAREZ-MARIN, A. (2013). "Análisis de las capacidades emprendedoras potenciales y efectivas en alumnos de centros de educación superior". Journal of technology management \& innovation, vol. $8, \mathrm{n}^{\circ} 1$, pp. 65-75.

CANO, C., GARCÍA, J., y GEA, A. (2003). Actitudes emprendedoras y creación de empresas en estudiantes universitarios. Universidad de Almería.

CASERO-RIPOLLÉS, A., IZQUIERDO-CASTILLO, J., y DOMÉNECH-FABREGAT, H. (2016). "The Journalists of the Future Meet Entrepreneurial Journalism: Perceptions in the classroom". Journalism Practice, vol. 10, nº 2, pp. 286-303.

(c)) EY-NC-ND 2016, Universitat Politècnica de València

Congreso In-Red (2016) 
Cooperación, creatividad y emprendimiento para el desarrollo de competencias. Una experiencia docente en Periodismo.

CAZAU, P. (2004). "Estilos de aprendizaje: el modelo de los cuadrantes cerebrales". En GÓMEZ, J. (coord.). Neurociencia cognitiva y educación. Serie: materiales de postgrado. Lambayeque (Perú): FACHSE. UNPRG.

ESTRADA, M., MONFERRER, D., SEGARRA, M., y MOLINER, M. A. (2014). "El trabajo cooperativo en base al modelo del cerebro total" en REDMARKA. Revista Digital de Marketing Aplicado, 12, pp. 87-112.

GARCÍA, A.J., y TROYANO, Y. (2010). "Aprendizaje cooperativo en personas mayores universitarias" en Revista Interamericana de Educación de Adultos, vol. 32, n 1, pp. 1-15.

GARCÍA, R., TRAVER, J.A., y CANDELA, I. (2001). Aprendizaje cooperativo. Fundamentos, características y técnicas. Madrid: Editorial CCS.

GIL, C., ALÍAS, A., y MONTOYA, M.D.G. (2006). "Cómo mezclar diferentes metodologías docentes para motivar e impulsar a un mayor número de alumnos", VI Jornadas de Aprendizaje Cooperativo. Barcelona.

HATTEN, T. (1993). Student attitude toward entrepreneurship as affected by participation in small business institute program. Michigan: UMI Dissetation Services.

HERRMANN, S.L.M. (1989). The creative brain evolution. Búfalo: Brain Books.

JIMÉNEZ, C.A. (2006). Diagnóstico teoría del cerebro total. Pereira: Magisterio.

JOHNSON, D.W., y JOHNSON, R.T. (1989). "Cooperative learning: What special education teachers needs to know”, Pointer, vol. 33, n² 2, pp. 5-10.

KRUEGER , N., y BRAZEAL, D. (1994). "Entrepreneurial potential and potential entrepreneurs". Entrepreneurship Theory and Practice, pp. 91-104.

LAUKKANEN, M. (2000). "Exploring alternative approaches in high-level entrepreneurship education: creating microchanisms for endogenous regional growth". Entrepreneurship \& Regional Development, pp 25-48.

LOLI A., DEXTRE, E., DEL CARPIO, J., y LA JARA, E. (2010). Actitudes de creatividad y emprendimiento en estudiantes de la Universidad Nacional de Ingeniería y su relación con algunas variables sociodemográficas. Perú: Universidad Nacional Mayor de San Marcos.

MARKHAM, T., MERGENDOLLER, J., LARMER, J., y RAVITZ, J. (2003). Project Based Learning Handbook. Buck Institute for Education.

MORALES PARRAGUÉ, M. (2009). El clima de emprendimiento, un determinante clave en la intención emprendedora de los estudiantes de Escuelas de Negocio. Universidad de Chile. Santiago: Momento Cero.

OVEJERO BERNAL, A. (1990). El aprendizaje cooperativo: una alternativa eficaz a la enseñanza tradicional. Barcelona: Promociones y Publicaciones Universitarias, S. A.

TRAVER, V.J. (2011). "Una experiencia de aprendizaje cooperativo basado en proyectos en una asignatura de diseño de interfaces de usuario Título del artículo entrecomillado" en ReVisión, vol. 4, $n^{\circ} 2$.

VILES, E., ZÁRRAGA-RODRÍGUEZ, M., y JACA, C. (2013). "Herramienta para evaluar el funcionamiento de los equipos de trabajo en entornos docentes", Intangible Capital, vol. 9, $\mathrm{n}^{\circ} 1$, pp.281-304.

(cc) EY-NC-ND 2016, Universitat Politècnica de València 